\title{
The anti-dengue virus properties of statins may be associated with alterations in the cellular antiviral profile expression
}

\author{
OWEN LLOYD BRYAN-MARRUGO ${ }^{1}$, DANIEL ARELLANOS-SOTO ${ }^{1}$, \\ AUGUSTO ROJAS-MARTINEZ ${ }^{1}$, HUGO BARRERA-SALDAÑA ${ }^{1}$, JAVIER RAMOS-JIMENEZ ${ }^{2}$, \\ ROMAN VIDALTAMAYO ${ }^{3}$ and ANA MARÍA RIVAS-ESTILLA ${ }^{1}$ \\ ${ }^{1}$ Department of Biochemistry and Molecular Medicine; ${ }^{2}$ Infectious Disease Service, School of Medicine, \\ Autonomous University of Nuevo León; ${ }^{3}$ Department of Basic Sciences, University of Monterrey, \\ Monterrey, Nuevo León 64460, México
}

Received June 10, 2015; Accepted February 12, 2016

DOI: $10.3892 / \mathrm{mmr} .2016 .5519$

\begin{abstract}
Dengue virus (DENV) susceptibility to cholesterol depleting treatments has been previously reported. There are numerous questions regarding how DENV seizes cellular machinery and cholesterol to improve viral production and the effect of cholesterol sequestering agents on the cellular antiviral response. The aim of the present study was to evaluate the mechanisms involved in the negative regulation of DENV replication induced by agents that diminish intracellular cholesterol levels. Cholesterol synthesis was pharmacologically (fluvastatin, atorvastatin, lovastatin, pravastatin and simvastatin treatment) and genetically (HMGCR-RNAi) inhibited, in uninfected and DENV2-infected hepatoma Huh-7 cells. The cholesterol levels, DENV titer and cellular antiviral expression profile were evaluated. A reduction in the DENV titer, measured as plaque forming units, was observed in DENV-infected cells following $48 \mathrm{~h}$ treatment with $10 \mu \mathrm{M}$ fluvastatin, $10 \mu \mathrm{M}$ atorvastatin, $20 \mu \mathrm{M}$ lovastatin and $20 \mu \mathrm{M}$ simvastatin, which achieved 70, 70, 65 and 55\% DENV2 inhibition, respectively, compared with the untreated cells. In addition, the cytopathic effect was reduced in the statin-treated DENV-infected cells. Statins simultaneously reduced cholesterol levels at $48 \mathrm{~h}$, with the exception of DENV2 infected cells. Genetic inhibition of cholesterol synthesis was performed using RNA interference for 3-hydroxy-3-methylglutaryl-CoA reductase (HMGCR-siRNA), which indicated a slight
\end{abstract}

Correspondence to: Dr Ana María Rivas-Estilla, Department of Biochemistry and Molecular Medicine, School of Medicine, Autonomous University of Nuevo León, 1000 Calle Gonzalitos, Mitras Centro, Monterrey, Nuevo León 64460, México

E-mail: amrivas1@yahoo.ca

Abbreviations: DENV, dengue virus; FLU, fluvastatin; ATO, atorvastatine; LOV, lovastatin; PRE, pravastatin; SIM, simvastatin; MOI, multiplicity of infection; RT-PCR, reverse transcription polymerase chain reaction; $\mathrm{PFU}$, plaque forming units

Key words: dengue virus, statins, antiviral response, expression profile, cholesterol, 3-hydroxy-3-methyl-glutaryl-CoA reductase reduction in DENV2 titer at 48 h post-infection, however, with no significant reduction in cholesterol levels. In addition, DENV2 infection was observed to augment the intracellular cholesterol levels in all experimental conditions. Comparison between the cellular antiviral response triggered by DENV2 infection, statin treatment and HMGCR-siRNA in infected, uninfected, treated and untreated Huh7 cells, showed different expression profiles for the antiviral genes evaluated. All downregulating cholesterol agents evaluated reduced the expression of genes associated with cellular immune and pro-inflammatory responses. These results indicate that statin-mediated downregulation of DENV2 infectious particles number is independent of cholesterol levels and it is partially mediated by the modulation of the cellular antiviral profile.

\section{Introduction}

Dengue is an important mosquito borne diseases in tropical and sub-tropical regions. In recent decades, the infection has spread significantly, reaching greater than 50 million cases per year (1). Dengue disease is caused by the dengue virus (DENV), which belongs to the flaviviridae family, and is an enveloped virus formed by a single stranded RNA genome and includes 4 serotypically different viruses (DENV-1 to 4) (2). Infection with any of the four serotypes results in similar pathological manifestations, which range from sub-clinical discomfort in approximately $30 \%$ of cases, dengue fever in $70 \%$ of cases to dengue hemorrhagic fever and significant mortality in $1 \%$ of cases $(3,4)$. The pathogenesis of DENV infection remains to be fully understood.

There is no specific therapy to treat the disease, and clinical management is limited to supportive care. Certain reports have indicated that cholesterol and lipid synthesis pathways are associated with DENV infection, and in addition, DENV susceptibility to cholesterol depleting treatments has been reported (5). Furthermore, in vitro studies have demonstrated the importance of the cholesterol pathway in viral fusion, replication and assembly. However, conflicting reports have indicated that the addition of cholesterol upon viral binding and attachment blocks the entry of the flavivirus into the cell and impairs viral replication $(6,7)$. It remains unclear how viruses 
use cellular machinery and molecules including cholesterol, to support viral production. It has been reported that genetic inhibition of cholesterol biosynthesis (siRNA against mevalonate decarboxylase) reduced DENV replication in an in vitro replicon (8). Another previous study demonstrated that cholesterol present in the viral envelope is necessary for successful infection, however, cell membrane cholesterol depletion had no effect on DENV infectivity towards several animal and human cell lines (9). These apparently conflicting results suggest that DENV binding and attachment is independent of cell membrane cholesterol but dependent on viral particle cholesterol levels.

Alternatively, genetic and pharmacological inhibition of cholesterol synthesis has been indicated to impair the late stages of the DENV cycle, including viral replication, particle assembly and viral budding $(10,11)$. However, the mechanisms of DENV interaction with cholesterol rich compartments in the cell remain to be fully understood. The inhibitory properties of cholesterol modifying agents towards DENV appear to be orchestrated by additional mechanisms involving the modulation of host genes. Statins are a group of drugs that were initially developed to reduce lipid levels. However, previous reports have raised considerable interest in the additional properties of statins, including anti-inflammatory effects at the endothelium and antiviral effects against dengue and hepatitis $\mathrm{C}$ viruses (12). It is possible that such antiviral effects may additionally be mediated by the regulation of cellular metabolic pathways, such as the antiviral profile of the cell. Previous studies have connected pathogens, cholesterol metabolism and cell antiviral profile (13-15). Therefore, the aim of the present study was to elucidate the mechanisms involved in the negative regulation of DENV replication induced by agents that reduce intracellular cholesterol levels.

\section{Materials and methods}

Cell culture and viral strains. The Huh7 hepatocyte derived carcinoma cells were used for the inhibition experiments. Cells were grown in advanced Dulbecco's modified Eagle medium (ADMEM; Thermo Fisher Scientific, Inc., Waltham, MA, USA) supplemented with $2 \mathrm{mM}$ glutamine, $5 \%$ fetal bovine serum (FBS), penicillin $\left(5 \times 10^{4} \mathrm{U} / \mathrm{ml}\right)$-streptomycin $(50 \mu \mathrm{g} / \mathrm{ml}), 1 \mathrm{ml} / 1$ amphotericin $\mathrm{B}$, non-essential amino acids, at $37^{\circ} \mathrm{C}$ in a $5 \% \mathrm{CO}_{2}$ atmosphere. Additionally, baby hamster kidney [BHK-21; American Type Culture Collection (ATCC); Manassas, VA, USA] cells were used for the DENV titer assessment. BHK-21 cells were cultured on minimum essential medium (MEM; Sigma-Aldrich, St. Louis, MO, USA), $5 \%$ FBS, penicillin $\left(5 \times 10^{4} \mathrm{U} / \mathrm{ml}\right)$-streptomycin $(50 \mu \mathrm{g} / \mathrm{ml})$, $1 \mathrm{ml} / 1$ anphotericin $\mathrm{B}$ and $2 \mathrm{mM}$ glutamine, non-essential amino-acids, at $37^{\circ} \mathrm{C}$ in a $5 \% \mathrm{CO}_{2}$ atmosphere. $\mathrm{C} 6 / 36 \mathrm{HT}$ cells (from Aedes albopictus and adapted to grow at $34^{\circ} \mathrm{C}$ ), were grown at $34^{\circ} \mathrm{C}$ in MEM (Thermo Fisher Scientific, Inc., Waltham, MA, UAS), supplemented with 7\% FBS, non-essential amino acids, MEM vitamin solution (Sigma-Aldrich), $0.370 \mathrm{~g} / 1$ sodium bicarbonate, $50 \mathrm{U} / \mathrm{ml}$ of penicillin and $50 \mu \mathrm{g} / \mathrm{ml}$ of streptomycin. The dengue- 2 New Guinea $\mathrm{C}$ virus (GenBank M29095) was propagated in C6/36 insect cells (ATCC CRL-1660 ${ }^{\mathrm{TM}}$ ). Briefly, C6/36 HT cells grown in a $175 \mathrm{~mm}^{2}$ flask to $\sim 85 \%$ confluence at $34^{\circ} \mathrm{C}$, were infected at a multiplicity of infection (MOI) of 1 for $2 \mathrm{~h}$ at $34^{\circ} \mathrm{C}$. Subsequently, fresh medium was added and the infection was permitted until cytopathic effects were observable at $34^{\circ} \mathrm{C}$. Cells were then lysed by 20 strokes using a glass pestle and tube at $4^{\circ} \mathrm{C}$, and lysates were centrifuged at $1,000 \times \mathrm{g}$ at $4^{\circ} \mathrm{C}$ to eliminate cellular debris. DENV particles were then precipitated in a 1 in 4 dilution of polyethylene glycol in $2 \mathrm{M} \mathrm{NaCl}$ overnight. The solution was centrifuged at $6,000 \mathrm{rpm}$ for $1 \mathrm{~h}$ to separate DENV particles, prior to re-suspension in GNTE buffer (200 mM glycine, $100 \mathrm{mM} \mathrm{NaCl}, 1 \mathrm{mM}$ EDTA, $50 \mathrm{mM}$ Tris-base at $\mathrm{pH} 7.5)$ and aliquoted and stored at $-70^{\circ} \mathrm{C}$ until use. For DENV ultraviolet (UV) light inactivation, viral preparations with a titer of $1 \times 10^{6}$ plaque forming units $(\mathrm{PFU}) / \mathrm{ml}$ were placed on ice and irradiated with a UV lamp for $1 \mathrm{~h}$. The irradiated virus preparations were titrated by plaque assay to confirm viral inactivation.

Dengue virus titration. Dengue virus titers were determined by plaque assays in confluent monolayers of BHK-21 cells grown at 80,000 cells/well. BHK-21 cells were inoculated with 10-fold serial dilutions of supernatants from each experimental condition [lovastatin (LOV), pravastatin (PRA), atorvastatin (ATO), fluvastatin (FLU) and simvastatin (SIM) purchased from Sigma Aldrich, and HMGCR RNAi] when monolayers reached $80-90 \%$ confluence. Following 2 h of viral adsorption, the monolayers were overlaid with $1 \mathrm{ml}$ of MEM containing $1 \%$ carboximethil-cellulose (Sigma Aldrich), 0.5\% FBS and $2 \mathrm{mM} \mathrm{L}$ glutamine. The cultures were incubated at $37^{\circ} \mathrm{C}$ for 5 days and the the PFU counted following staining with $0.5 \%$ naphtol-blue-black (Sigma Aldrich).

Cytotoxicity assay. The cytotoxicity of statins was measured using a 3-(4,5-dimethylthiazol-2-yl)-2,5-diphenyltetrazolium bromide (MTT) assay (Roche Diagnostics, Basel, Switzerland) based on the reduction of the tetrazolium salt, MTT, in the presence of an electron-coupling reagent. Cells grown in a 96-well plate at 20,000 cells/well, the tissue culture plate were treated for the indicated periods and then incubated with the MTT solution for $\sim 4 \mathrm{~h}$. Following this incubation period, water-insoluble formazan crystals were formed, they were solubilized using $10 \%$ SDS in $0.01 \mathrm{M} \mathrm{HCl}$, the formazan dye was quantified using a scanning multi-well plate reader at $585 \mathrm{~nm}$.

Pharmacological inhibition of DENV infection. For all antiviral profile experiments, cells were treated for $48 \mathrm{~h}$ in the same conditions Huh-7 cells were incubated and $24 \mathrm{~h}$ later were infected with DENV-2 at an MOI of 1. Following $12 \mathrm{~h}$ of infection, the cells were treated with LOV $(20 \mu \mathrm{M})$, PRA $(50 \mu \mathrm{M})$, ATO $(10 \mu \mathrm{M}), \operatorname{FLU}(10 \mu \mathrm{M})$ and SIM $(20 \mu \mathrm{M})$ (Sigma-Aldrich) at indicated concentrations, and then incubated for $48 \mathrm{~h}$. For the different treatments, Huh7 cells were plated, and one day later the media was replaced and the cells were treated with each statin, with this considered as time zero. At the end of the incubation, the supernatants were collected to measure the PFU for each treatment in order to quantify the DENV particles and the cholesterol levels using Cayman's Cholesterol Fluorimetric Assay kit (Cayman Chemical, Ann Arbor, MI, USA) according to the manufacturer's instructions. All determinations were performed in triplicate. 
RNA interference (RNAi) assay to inhibit 3-hydroxy-3-methylglutaryl-CoA reductase (HMGCR) expression. A pre-validated heterogeneous mixture of 21-22 base pair short interfering RNAs (siRNA) that specifically inhibits HMGCR expression (cat. no. AM51331; Ambion; Thermo Fisher Scinetific, Inc.), were used according to the manufacturer's instruction. Huh7 cells $\left(5 \times 10^{5}\right)$ were seeded on 12-well plates in ADMEM containing 2\% FBS, $1 \%$ nonessential amino acids, $100 \mathrm{U} / \mathrm{ml}$ penicillin $\mathrm{G}$ and $100 \mathrm{mg} / \mathrm{ml}$ streptomycin. Following seeding, the cells were transfected with serum-free DMEM and $100 \mathrm{mM}$ siRNA directed against HMGCR (siRNA-HMGCR). Cells were at 50-60\% confluence at the time of transfection and $24 \mathrm{~h}$ later were incubated in the presence or absence of DENV-2, for $2 \mathrm{~h}$. siRNAs were transfected at a final concentration of $100 \mathrm{nM}$, using siPORT Lipid Transfection Agent (Ambion; Thermo Fisher Scientific, Inc.). Silencer negative control siRNA (100 nM; cat. no. AM4611; Ambion; Thermo Fisher Scientific, Inc.) and untransfected cells containing siPORT Lipid Agent alone were used as negative controls, Select Silencer GAPDH Positive Control siRNA (cat no. AM4390849; Ambion; Thermo Fisher Scientifc, Inc.) was also used. Cells were incubated and the infection was allowed to progress for $48 \mathrm{~h}$. The statin concentrations were selected based on previous toxicity assessment.Statins stock solutions were diluted with dimethyl sufloxide (DMSO) and adjusted to $0.1 \%$ final concentration for all statins. DMSO was used as control for the statin treatment and UV-inactivated DENV2 for the mock infection. Following each incubation, total RNA was extracted and reverse transcribed to cDNA using a high-capacity cDNA archive kit (Applied Biosystems; Thermo Fisher Scientific, Inc.). From 100 ng of cDNA, quantitative polymerase chain reaction (qPCR) was performed to quantify HMGCR-mRNA levels, using the following primers: P1: 5'-GACGCAACCTTTATATCCGTTT-3' and P2: 5'-TTT TGAAAGTGCTTTCTCTGTACC-3'. For each PCR reaction, $10 \mu l$ of SYBR Green PCR-Master mix (Invitrogen; Thermo Fisher Scientific, Inc.), $1 \mu \mathrm{l}$ of each primer $10 \mu \mathrm{M}$, and $5 \mu \mathrm{l}$ of cDNA diluted in RNase-free water were added. Thermal cycling conditions were as follows: Initial step at $50^{\circ} \mathrm{C}$ for $2 \mathrm{~min}$, then $95^{\circ} \mathrm{C}$ for $10 \mathrm{~min}$, followed by 35 cycles of $95^{\circ} \mathrm{C}$ for $15 \mathrm{sec}$ and $60^{\circ} \mathrm{C}$ for $60 \mathrm{sec}$. Fluorescence was monitored at the annealing step, and amplification plots were generated. GAPDH expression was used to normalize the RNA concentration. For GAPDH-RNA quantification, a GAPDH (20X) assay was used (Applied Biosystems; Thermo Fisher Scientific, Inc.) according to the manufacturer's specifications. In parallel, the supernatant was used to measure the PFUs to quantify the DENV particles. HMGCR-mRNA levels were normalized based on the ratio of HMGCR/ACT mRNA. Data are expressed as the relative fold levels of HMGCR-RNA to the cells transfected with silencer negative control siRNA, which was set at 1.0. The mRNA levles were calculated using the $2^{-\Delta \Delta \mathrm{Cq}}$ method.

Cellular cholesterol determination. Huh-7 cells grown in 6 well plates were infected and treated as described above. Subsequently, cells were washed 3 times with phosphate-buffered saline and lysed with $250 \mu 1$ lysis buffer RSB-NP40 (1.5 mM MgCl2, 10 mM Tris- $\mathrm{HCl} \mathrm{pH} 7.5,10 \mathrm{mM} \mathrm{NaCl}$, $1 \%$ IGEPAL) at $48 \mathrm{~h}$. Cells were homogenized on ice by 10 strokes in a glass pestle and tube. Subsequently, the cholesterol levels were measured using Fluorimetric Cholesterol assay kit (Cayman Chemical Company, Ann Arbor, MI, USA). Determinations were performed according to the manufacturer's instructions.

$R T^{2}$ profiler PCR arrays. $\mathrm{RT}^{2}$ Profiler PCR Arrays for antiviral pathway assessment (cat. no. PAHS-122Z) were used to analyze RNA expression (Qiagen $\mathrm{GmbH}$, Hilden, Germany). The $\mathrm{RT}^{2}$ Profiler PCR Arrays in 96-well plates contained primer assays for 84 antiviral pathway genes and 5 housekeeping genes. In addition, one well contains a genomic DNA control, 3 wells contain reverse-transcription controls, and 3 wells contain positive PCR controls. Total RNA from samples was extracted using an RNeasy Plus Mini kit (Qiagen $\mathrm{GmbH}$ ) following the manufacturer's instructions. Briefly, samples were first lysed and homogenized in a highly denaturing guanidine-isothiocyanate-containing buffer, which immediately inactivates RNases to ensure isolation of intact RNA. The lysate was then passed through a gDNA eliminator spin column. This column, in combination with the optimized high-salt buffer, allows efficient removal of genomic DNA. Ethanol was added to the flow-through to provide appropriate binding conditions for RNA, and the sample was then applied to an RNeasy spin column, where total RNA binds to the membrane and contaminants were efficiently washed away. High-quality RNA was then eluted in $30 \mu \mathrm{l}$ water. Subsequently, the experimental RNA samples were converted into first-strand cDNA using the RT ${ }^{2}$ First Strand kit (Qiagen $\mathrm{GmbH}$ ). The cDNA was then mixed with the $\mathrm{RT}^{2} \mathrm{SYBR}$ Green Mastermix (Qiagen $\mathrm{GmbH})$. PCR was performed according to the manufacturer's protocol and the relative expression was determined using the $2^{-\Delta \Delta C q}$ method. An Applied Biosystem 7500 Real-Time Cycler (Thermo Fisher Scientific, Inc) was used, and qPCR 7500 software, version 2.0.1 (Thermo Fisher Scientific, Inc.) and $\mathrm{RT}^{2}$ Profiler PCR Array Data Analysis software, version 3.5 (Qiagen $\mathrm{GmbH}$ ) were used for data analysis.

Statistical analysis. All variables were tested in triplicate, and experiments were repeated a minimum of three times. Values are expressed as the mean \pm standard deviation. One-way analysis of variance was used to test for differences between groups, followed by Tukey's honest significant difference test. All data analysis was performed using SPSS (SPSS, Inc., Chicago, IL, USA). P $<0.05$ was considered to indicate a statistically significant difference.

\section{Results}

Viability of hepatoma Huh7 cells treated with different statins. First, it was determined whether statins are able to induce a cytotoxic effect on treated cells. Huh7 cells were exposed to four different concentrations of each statin, LOV, PRA, ATO, FLU and SIM (10-1,000 $\mu \mathrm{M})$ and incubated for 24-72 h, following which the total cell count and viability determinations were performed using an MTT assay. Fig. 1A indicates that following $72 \mathrm{~h}$ of treatment, no significant difference in cell viability was observed between the untreated (100\% viability) and treated cell lines at concentrations below $50 \mu \mathrm{M}$ for each statin (85-95\%; P>0.05; Fig. 1A). However, 
cells treated with higher concentrations (over $100 \mu \mathrm{M}$ ) showed lower cell survival following $72 \mathrm{~h}$ of exposure with the exception of PRA, which did not lead to a reduction in cell survival at that concentration. Based on these results, concentrations lower than $50 \mu \mathrm{M}$ were selected for each statin treatment in the subsequent experiments.

Statins exert differential in vitro antiviral effects against $D E N V$. The present study sought to evaluate whether the modulation of the cholesterol synthetic pathway would have an impact on DENV 2 infection. Statins target the HMGCR enzyme, which is the rate-controlling enzyme of the cholesterol synthesis pathway. To evaluate the effect of the five different statins on the DENV viral cycle, Huh-7 cells were infected with DENV-2 (New Guinea strain) at MOI 1 for $24 \mathrm{~h}$. Subsequently, the infected cells were treated for $48 \mathrm{~h}$ with $20 \mu \mathrm{M}$ LOV, $50 \mu \mathrm{M}$ PRA, $10 \mu \mathrm{M}$ ATO, $10 \mu \mathrm{M}$ FLU and $20 \mu \mathrm{M}$ SIM. Following treatment, the media supernatant was collected and used to evaluate the viral titer using a PFU assay, and cells were observed for the assessment of the cytopathic effect. It was observed that the majority of the statins reduced the DENV titer at $48 \mathrm{~h}$ compared with the untreated cells $(\mathrm{P}<0.001$; Fig. 1B). In addition, a differential capacity to reduce the DENV titer was observed between the tested statins. FLU exerted greater than $75 \%$ inhibition on the mature infectious viruses released into the supernatants, followed by ATO with $72 \%$ and LOV with $68 \%$ inhibition compared with the untreated infected cells. SIM and PRA exhibited a moderate effect on the viral titer at the selected concentrations (Fig. 1B). These results indicate that statins exert significant inhibition on DENV2 titers suggesting that the dengue viral cycle is cholesterol dependent. At their maximum non-cytotoxic concentrations, the statins had different antiviral profiles against DENV2 in the cell culture system used (Fig. 1B). The results for the cytopathic effect assessment on LOV, ATO and PRA treated cells supported those of the DENV2 viral titer (data not shown).

DENV2 infection upregulates cholesterol synthesis. It has been reported that cholesterol is associated with sub-cellular compartments in which viral replication and maturation occur, however, previous studies have reported that certain stages of cellular DENV infection are independent of cholesterol (16). Therefore, the present study aimed to investigate whether the observed antiviral properties of statins correlated with cholesterol levels in infected cells. A flourometric assay was performed to quantify the inhibition of cholesterol by statins on infected and uninfected Huh-7 cells. Uninfected and infected cells with DENV-2 (MOI 1) were cultured for $24 \mathrm{~h}$ and then treated with the statins at the previously indicated concentrations. Cells were then harvested at 0,24 and $48 \mathrm{~h}$ following treatment and the intracellular cholesterol levels were measured. A moderate effect of the statins in reducing cholesterol levels was observed in the treated uninfected cells (LOV, 30\% ATO, 15\% PRA, $35 \%$ FLU and 25\% SIM compared with the untreated cells) $(\mathrm{P}<0.05$; Fig. 1C) with a greater effect at $48 \mathrm{~h}$ upon treatment. Contrary to expectations, the antiviral effect of statins may be contributed to both lowering of cholesterol and unknown cellular mechanisms.
Notably, it was observed that the cholesterol levels were greater in the DENV-infected cells compared with the un-infected cells, suggesting that DENV replication is able to modulate and increase cholesterol production $(\mathrm{P}<0.01$; Fig. 1C). Also, it was observed that in DENV-infected cells treated with the different statins the cholesterol levels were increased, compared with the untreated infected cells.

HMGCR-specific RNAi reduces DENV titer. To further investigate whether the inhibition of cholesterol is associated with a reduction of the DENV titer, the effects of HMGCR-specific siRNA were examined in Huh7 cells in the presence or absence of DENV-2 infection. HMGCR-specific siRNA was transfected into Huh7 cells, incubated for $24 \mathrm{~h}$, then cells were infected with DENV-2 for $1 \mathrm{~h}$ and incubated for $48 \mathrm{~h}$ further. Subsequently, the cells and supernatant were collected and HMGCR-mRNA levels, cholesterol and DENV titer were quantified. This indicated that the introduction of the HMGCR specific siRNA reduced the expression of HMGCR-mRNA to $\sim 45 \%$ in Huh7-infected cells ( $\mathrm{P}<0.05$; Fig. $2 \mathrm{~A})$. In addition, the cholesterol levels were reduced in the uninfected cells at $48 \mathrm{~h}$ post-infection in all conditions $(\mathrm{P}<0.05$; Fig. 2B). Regarding the effect of HMGCR siRNA on the DENV titer, genetic inhibition of HMGCR reduced the viral titer by $45 \%$ compared with the control cells $(\mathrm{P}<0.05$; Fig. $2 \mathrm{C})$.

A control siRNA directed against the GAPDH gene was observed to reduce GAPDH-mRNA (data not shown) and did not have any significant effects on the expression levels of HMGCR-mRNA (Fig. 2A), DENV titer (Fig. 2B) cholesterol levels (Fig. 2C) in uninfected and infected cells. A scrambled siRNA sequence was used as a control of specificity (Fig. 2A). It is of note that partial knockdown of HMGCR expression was able to significantly reduce cholesterol levels only in uninfected cells; however, in DENV-infected cells reduction in cholesterol levels was not significant. As aforementioned, DENV may be inducing unknown mechanisms, which lead to the increase of cholesterol levels. Together these results suggest that partial genetic inactivation of HMGCR reduced DENV titer; however did not necessarily modify cholesterol in DENV-infected cells.

LOV and HMGCR-specific siRNA treatment modify the antiviral cell response in DENV2-infected cells. Although these results suggest that negative regulation of DENV replication mediated by statins and HMGCR genetic blockage, cholesterol levels were not affected in infected cells; therefore, it is likely that other mechanisms are also involved in this phenomenon. To further investigate these mechanisms, the cellular antiviral response profile of statin-treated and DENV-infected cells was assessed. Huh-7 cells were infected with DENV2 at MOI 1, then treated with $20 \mu \mathrm{M}$ LOV for $24 \mathrm{~h}$, washed and the infection left to progress for $48 \mathrm{~h}$. Un-infected untreated Huh-7 cells were used as a control.

Total RNA was extracted and reverse transcribed to cDNA for PCR-array analysis using a Qiagen PCR-Array platform. A 3 -fold alteration in gene expression was considered significant. The results indicate that following infection with DENV2, a number of genes associated with the cellular antiviral response were upregulated on infection compared with the uninfected Huh-7 cells. Table I presents all the altered genes 
Table I. Genes differentially expressed following HMGCR knockdown or LOV treatment in DENV-infected cells.

A, HMGCR knockdown

\begin{tabular}{|c|c|c|c|c|c|}
\hline \multirow[b]{2}{*}{ Symbol } & \multicolumn{5}{|c|}{ Fold change } \\
\hline & DENV2+ & HMGCR+ & $\begin{array}{c}\text { HMGCR+/ } \\
\text { DENV2+ }\end{array}$ & Nonsense+ & $\begin{array}{c}\text { Nonsense+/ } \\
\text { DENV2+ }\end{array}$ \\
\hline APOBEC $3 G$ & 2.5913 & 1.6792 & 1.2715 & 2.1757 & 1.0884 \\
\hline CASP1 & 0.8947 & 0.1482 & 1.4944 & 0.5314 & 0.8266 \\
\hline CASP10 & 1.1208 & 0.2921 & 1.1763 & 0.6859 & 0.6429 \\
\hline CASP8 & 1.8980 & 0.1283 & 1.0563 & 0.7463 & 0.7720 \\
\hline CCL3 & 2.8558 & 0.2629 & 0.9965 & 1.0633 & 0.9265 \\
\hline CCL5 & 3.4334 & 0.0615 & 0.5424 & 0.3904 & 0.6373 \\
\hline CXCL10 & 1.1153 & 0.2406 & 0.9339 & 0.5363 & 0.6072 \\
\hline CXCL11 & 0.7471 & 0.1264 & 1.036 & 0.3158 & 0.3702 \\
\hline CXCL9 & 0.6312 & 0.8372 & 0.9695 & 1.1206 & 0.3793 \\
\hline DAK & 2.5950 & 0.2479 & 2.9312 & 2.5551 & 2.8154 \\
\hline IL6 & 0.4649 & 0.7981 & 0.8367 & 0.3276 & 0.4456 \\
\hline IRAK1 & 1.6561 & 0.2766 & 4.5517 & 4.1872 & 3.7730 \\
\hline IRF7 & 1.6136 & 0.7975 & 0.7024 & 1.1784 & 3.1306 \\
\hline ISG15 & 2.7561 & 0.9373 & 1.4947 & 1.0859 & 1.5254 \\
\hline JUN & 3.7323 & 0.7412 & 2.1547 & 2.0135 & 1.8654 \\
\hline MAP2K1 & 3.4680 & 0.8739 & 1.5281 & 1.3052 & 1.4556 \\
\hline MX1 & 2.6361 & 0.9405 & 1.3409 & 1.2384 & 1.4264 \\
\hline MYD88 & 2.5260 & 1.1246 & 1.1519 & 0.6599 & 1.0037 \\
\hline NFKB1 & 3.0587 & 0.6116 & 1.0658 & 0.9385 & 0.8300 \\
\hline NLRP3 & 2.0192 & 1.3297 & 1.1711 & 0.7204 & 1.2132 \\
\hline NOD2 & 2.0192 & 1.3297 & 1.1711 & 0.7204 & 1.2132 \\
\hline OAS2 & 2.0192 & 1.3297 & 1.2084 & 0.7808 & 1.4042 \\
\hline SPP1 & 6.0050 & 1.0592 & 1.4141 & 0.8863 & 1.7468 \\
\hline STAT1 & 2.4066 & 0.6361 & 1.0925 & 0.8951 & 0.9486 \\
\hline TLR8 & 2.0192 & 1.3297 & 1.1711 & 0.7204 & 1.2132 \\
\hline TLR9 & 1.1410 & 0.7514 & 5.3502 & 0.8110 & 1.4939 \\
\hline TNF & 4.2555 & 1.2103 & 1.0659 & 2.4258 & 1.2316 \\
\hline TRADD & 2.4880 & 0.3227 & 1.715 & 2.4017 & 2.2466 \\
\hline TRAF3 & 2.3467 & 0.6484 & 1.1828 & 1.1444 & 1.1295 \\
\hline TRIM25 & 2.4894 & 0.435 & 1.153 & 1.3754 & 1.1828 \\
\hline
\end{tabular}

B, Lovastatin treatment

Fold change

\begin{tabular}{lccc}
\cline { 2 - 3 } Symbol & DENV2+ & LOV+ & LOV+/DENV2+ \\
\hline APOBEC3G & $\mathbf{2 . 5 9 1 3}$ & $\mathbf{0 . 3 2 2 2}$ & 0.5959 \\
CASP8 & 1.8980 & $\mathbf{0 . 4 2 2 9}$ & $\mathbf{0 . 4 5 5 6}$ \\
CCL3 & $\mathbf{2 . 8 5 5 8}$ & $\mathbf{0 . 2 0 9 4}$ & $\mathbf{0 . 4 3 6 6}$ \\
CCL5 & $\mathbf{3 . 4 3 3 4}$ & $\mathbf{0 . 2 2 2 2}$ & $\mathbf{0 . 1 5 0 7}$ \\
CXCL10 & 1.1153 & $\mathbf{0 . 1 7 0 9}$ & $\mathbf{0 . 3 2 4 5}$ \\
CXCL11 & 0.7471 & $\mathbf{0 . 2 9 6 9}$ & $\mathbf{0 . 1 4 6 9}$ \\
CXCL9 & 0.6312 & $\mathbf{0 . 2 1 1 5}$ & $\mathbf{0 . 3 1 8 2}$ \\
ISG15 & $\mathbf{2 . 7 5 6 1}$ & 0.8487 & 1.2345 \\
JUN & $\mathbf{3 . 7 3 2 3}$ & 1.4106 & $\mathbf{0 . 4 2 1 4}$ \\
MAP2K1 & $\mathbf{3 . 4 6 8 0}$ & 0.8560 & 0.5121 \\
MX1 & $\mathbf{2 . 6 3 6 1}$ & 0.9190 & 0.9391
\end{tabular}


Table IB. Continued.

Fold change

\begin{tabular}{lccc}
\cline { 2 - 4 } Symbol & DENV2+ & LOV + & LOV+/DENV2+ \\
\hline MYD88 & $\mathbf{2 . 5 2 6}$ & 1.1338 & 1.5763 \\
NFKB1 & $\mathbf{3 . 0 5 8 7}$ & 0.9303 & 0.7462 \\
NLRP3 & $\mathbf{2 . 0 1 9 2}$ & $\mathbf{0 . 4 7 6 5}$ & 1.0178 \\
NOD2 & $\mathbf{2 . 0 1 9 2}$ & $\mathbf{0 . 4 7 6 5}$ & 1.0178 \\
OAS2 & $\mathbf{2 . 0 1 9 2}$ & 1.7578 & 1.0373 \\
SPP1 & $\mathbf{6 . 0 0 5 0}$ & $\mathbf{0 . 2 8 5 8}$ & $\mathbf{0 . 3 6 1 9}$ \\
STAT1 & $\mathbf{2 . 4 0 6 6}$ & 0.9093 & 0.7034 \\
TLR8 & $\mathbf{2 . 0 1 9 2}$ & $\mathbf{0 . 4 7 6 5}$ & 1.0178 \\
TNF & $\mathbf{4 . 2 5 5 5}$ & $\mathbf{0 . 4 7 4 7}$ & 0.9264 \\
TRADD & $\mathbf{2 . 4 8 8 0}$ & 1.8014 & 0.8012 \\
TRAF3 & $\mathbf{2 . 3 4 6 7}$ & 1.3013 & 0.6837 \\
TRIM25 & $\mathbf{2 . 4 8 9 4}$ & 1.0330 & 0.5596 \\
ACTB & 0.9643 & $\mathbf{2 . 4 9 1 6}$ & 1.8144 \\
\hline
\end{tabular}

Bold print indicates upregulation, bold italic print indicates downregulation. HMGCR, 3-hydroxy-3-methyl-glutaryl-CoA reductase; LOV, lovastatin; DENV2, dengue virus type 2.

in each condition compared with the uninfected untreated cells and Fig. 3 shows a heat map representation for the genes modulated in each condition. For example, CCL3, CCL5 and ISG15 (an interferon stimulated gene) were upregulated in DENV2-infected cells. In addition, NFKB1, STAT1, TNF, TRADD and TRAF3, well known as pro-inflammatory genes, were upregulated in the infected cells.

Regarding the effect of LOV on uninfected Huh-7 cells, notable results were observed, with several genes up- and downregulated in the antiviral pathway. In contrast with the previously described results in infected cells without treatment, in LOV treated infected-cells, CCL5 and CCL3 were downregulated following statin treatment, while OAS2, a gene that encodes a protein involved in the innate immune response to viral infection, was upregulated greater than 3-fold.

Additionally, the effect of LOV treatment on Huh-7 cells infected with DENV2 was investigated. Notably, it was observed that in LOV treated cells, the majority of genes involved in the cellular antiviral response remained unaffected or were downregulated compared with the untreated DENV2-infected cells. Among the few upregulated genes was IL-15, which is involved in cell proliferation and the induction of natural killer cells. However, CCL5 and the interferon induced CXCL11 gene, which are involved in cellular pro-inflammatory responses and the chemotaxis of activated $\mathrm{T}$ cells, respectively, were downregulated.

Furthermore, using the same approach the effect of HMGCR-RNAi on the cellular antiviral profile of Huh-7 DENV2-infected cells was assessed. A similar effect was observed as in LOV treated cells, in particular, TLR9, known as a double-stranded RNA sensor, was upregulated greater than 4-fold compared with untreated DENV2-infected cells. Additionally, it was observed that the SPP1, TNF- $\alpha$ and CCL5 genes were downregulated, similar to LOV-treated cells.

\section{Discussion}

A systematic review of a number of studies evaluating the role of cholesterol and lipids in DENV infections raised the idea that the effect of cholesterol modulation upon DENV infection may be influenced by the nature of the inhibitory drug, the period of treatment, the cell line and the serotype or viral strain. Despite these variations, some assumptions can be summarized. In the present study, the effect of five different statins were investigated in order to observe their particular antiviral profiles. It was observed that four of the five statins, LOV, ATO, FLU and SIM, possess antiviral activity against DENV2. PRA was the least effective statin against DENV, which may be attributed to its poor liposolubility, which delays its entrance into cells.

To further clarify the DENV infection mechanism, cholesterol synthesis was genetically inhibited using HMGCR-siRNA in infected cells. This showed that enzyme blockage slightly reduced the viral titer, and indicates that DENV entry into the cells is complex and flexible, involving other host proteins and mechanisms.

A marked increase in intracellular cholesterol levels was observed in infected cells compared with the uninfected cells at $48 \mathrm{~h}$ post infection with DENV2, however, further analysis is required to clarify whether this alteration is due to a stimulation of cholesterol transport or its biosynthesis. These data support previous studies in which stimulation with HMGCR and cholesterol transport has been reported in DENV-infected cells $(17,18)$. For DENV, it has been reported that internal membrane rearrangement such as endoplasmic reticulum expansion and the formation of replication micro-domains, occurs in the early stages of the infection through the modulation of lipid and cholesterol transport, by the expression of viral genes (18). DENV protein expression specifically increased lipid raft formation and total 
A

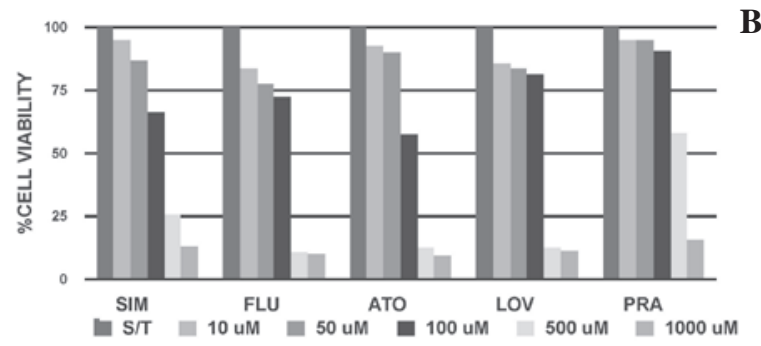

B

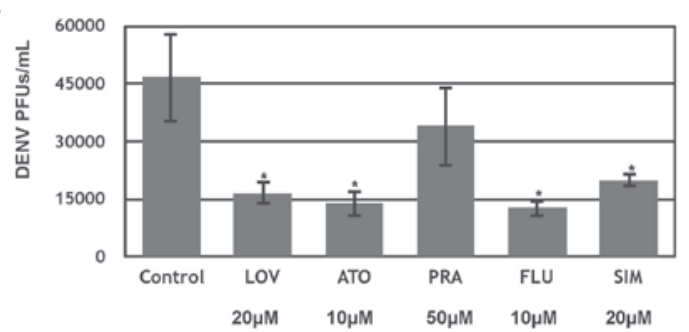

C

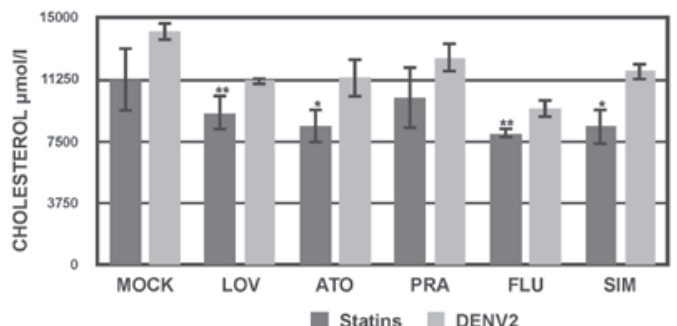

Figure 1. Effect of the pharmacological inhibition of cholesterol on DENV2 infection in HuH-7 cells. (A) Cytotoxic assessment of the effect of 72 h treatment with different concentrations of statins on Huh-7 cells, evaluated by 3-(4,5-dimethylthiazol-2-yl)-2,5-diphenyltetrazolium bromide assay. (B) Viral titer was evaluated by counting PFUs in the supernatant from each condition. (C) Intracellular cholesterol levels from cell extracts. ${ }^{*} \mathrm{P}<0.05$; ${ }^{* *} \mathrm{P}<0.01$. DENV2, dengue virus type 2; PFU, plaque forming units; LOV, lovastatin; ATO, atorvastatin; PRA, pravastatin; FLU, fluvastatin; SIM, simvastatin.

A

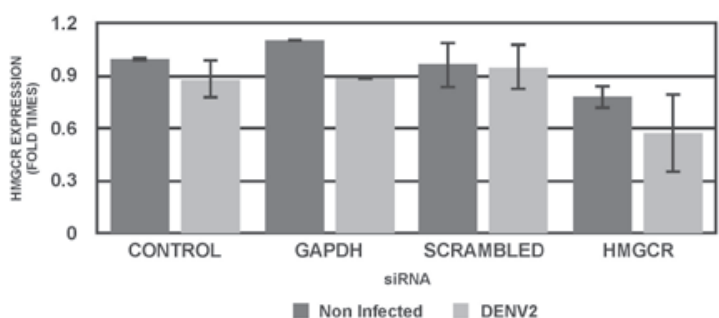

B

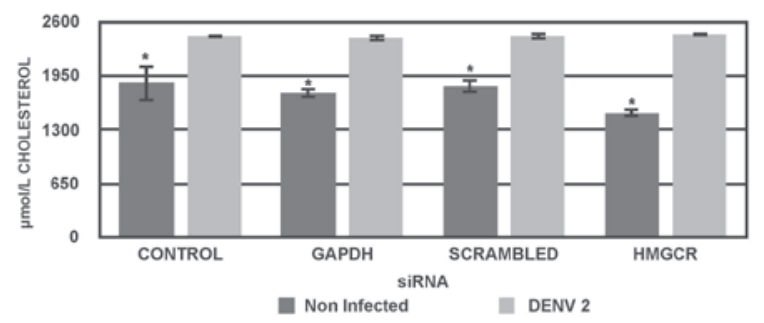

C

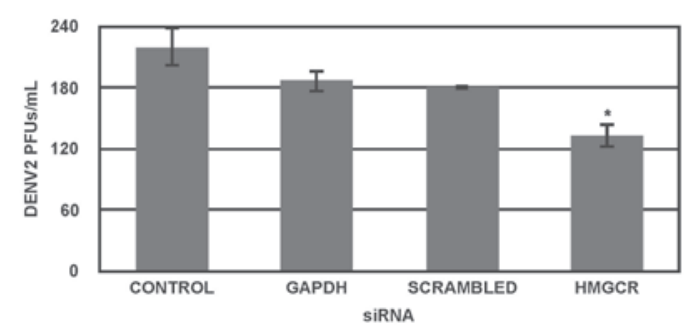

Figure 2. Effect of the genetic inhibition of cholesterol on DENV2 infection in HuH-7 cells. Huh-7 cells were transfected with HMGCR, GAPDH and scrambled RNAi and infected with DENV2. (A) HMGCR mRNA expression levels. (B) Intracellular cholesterol levels. (C) Viral titer. *P<0.05. DENV2, dengue virus type 2; HMGCR, 3-hydroxy-3-methyl-glutaryl-CoA reductase; GAPDH, glyceraldehyde 3-phosphate dehydrogenase; PFU, plaque forming units.

cholesterol levels at the early stages of infection by upregulating low-density lipoprotein particle uptake and promoting HMGCR activity (19).

In addition, the present study evaluated the modifications of the antiviral profile of Huh-7 cells as an alternative mechanism by which statins exert their antiviral activity. As expected, the cellular antiviral profile was altered by DENV2 infection. When the Huh-7 infected were compared with the uninfected cells, a number of genes were observed to be altered. The CCL3 and CCL5 genes were observed to be upregulated in the infected cells. In a previous study that evaluated DENV infection in mice, increased levels of these two chemokines were detected in the spleen and liver, and were indicative of severe dengue. Furthermore, in the same study, downregulation of the IL-6 gene was associated with a milder presentation of the disease (20). However in the present study, IL-6 was reduced in infected cells compared with the uninfected cells. In addition, ISG15, an interferon stimulated gene was observed to be upregulated in DENV2 infected cells, and this gene has been implicated in specific anti-DENV function via protein ISGylation, and its expression facilitates cellular antiviral responses to viral infection (14). In LOV treated cells, CCL5 and CCL3 were downregulated following treatment while OAS2, a gene that encodes a protein involved in the innate immune response to viral infection, was upregulated greater than 3 -fold. In 


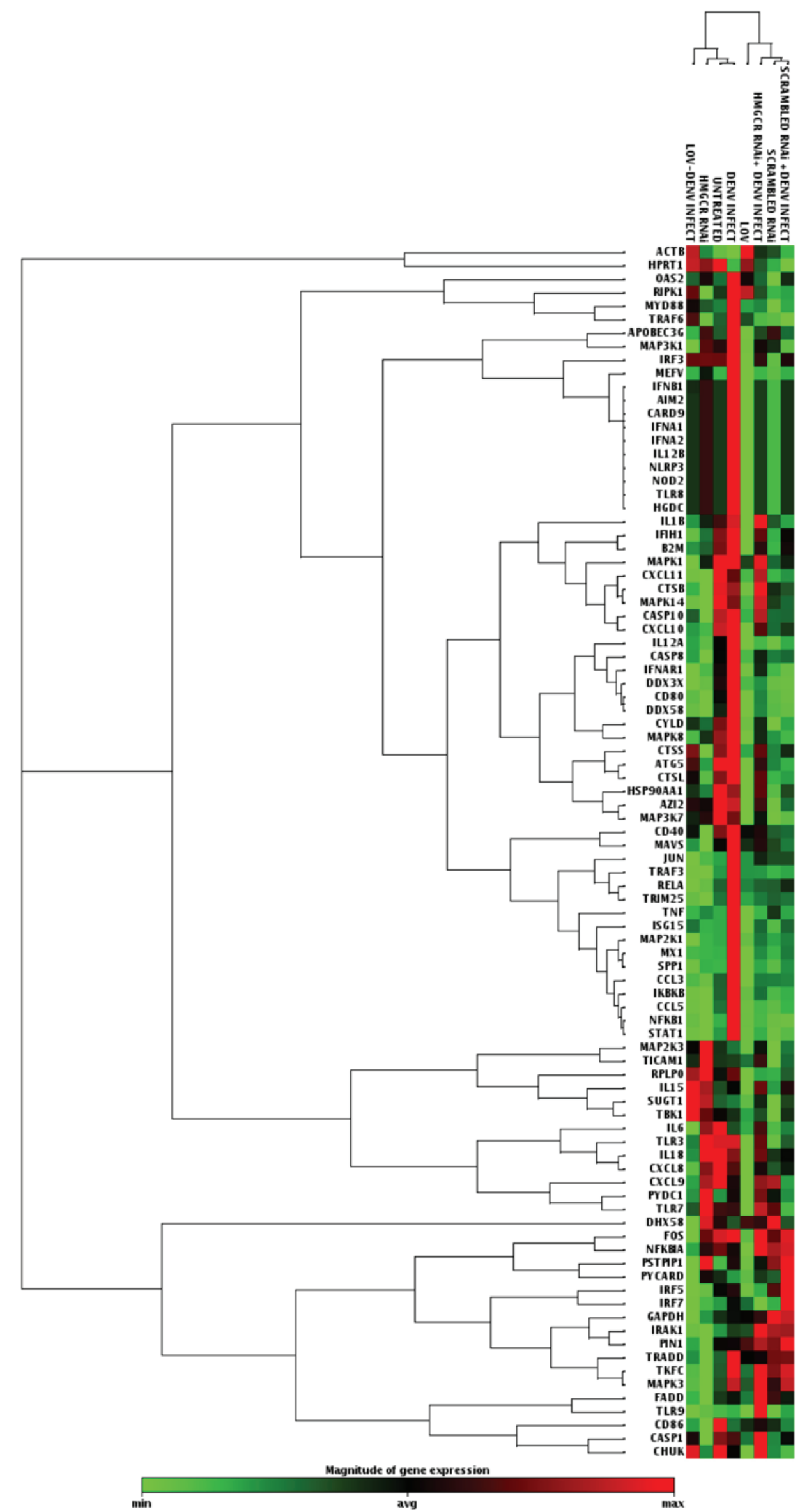

Figure 3. Clustergram of the differential expression of antiviral genes compared with the uninfected untreated cells. Huh-7 cells were transfected with HMGCR and scrambled RNAi, and treated with $20 \mu \mathrm{M} \mathrm{LOV}$, and the expression of cellular antiviral genes was evaluated by polymerase chain reaction array. HMGCR, 3-hydroxy-3-methyl-glutaryl-CoA reductase; RNAi, RNA interference; LOV, lovastatin; DENV, dengue virus. 
addition, TRADD, which encodes an apoptotic inhibitor protein, was upregulated in treated cells.

The results of the present study suggest that pharmacological and genetic inhibition of cholesterol may alleviate, to some extent, the pro-inflammatory state of the Huh7 hepatoma cells. To evaluate if an alternative pathway not associated with cholesterol was responsible for the antiviral effects of the statins and RNAi, infected cells were treated with LOV and HMGCR-siRNA. IL-15, involved in cell proliferation and the induction of natural killer cells, was upregulated by LOV treatment, however, this gene has been reported not to be stimulated by DENV infection, with only LOV treatment resulting in increased production (21). However, in uninfected LOV treated cells this gene was not altered and further studies are required to clarify its involvement in the DENV2 antiviral cellular response. By contrast, CCL5, SPP1 and TNF genes were downregulated by both LOV and HMGCR-siRNA treatments. Additionally, in contrast to what was expected, a number of genes in the cellular antiviral pathway were downregulated following LOV and RNAi treatment. This may render cells vulnerable to infection, and mean that cholesterol inhibition may reduce viral titer and attenuate antiviral gene expression. Another explanation for the downregulation of several antiviral genes in cholesterol diminished cells may be that this molecule participates in the synthesis and processing of these antiviral proteins, and that the blockade of cholesterol synthesis impairs their production.

In conclusion, a differential downregulation of DENV titer was observed to be induced by different statins. DENV2 infection resulted in a sustained increase in the levels of intracellular cholesterol following infection. However, whilst treatment with statins and HMGCR-siRNA reduced the viral titer, they did not do so via an effect on cholesterol levels. An alternative inhibitory mechanism was evaluated determining the antiviral profile modifications of the Huh7 cells following infection with DENV2, and LOV and HMGCR-siRNA treatment. It is not clear whether LOV and HMGCR-siRNA attenuation of cellular antiviral gene expression is due to DENV reduction through another undiscovered mechanism or via the stimulation of additional antiviral genes not evaluated in the present study. More detailed assessments are required to clarify the association between DENV replication and the role of cholesterol availability and cellular signaling pathways in infected cells.

\section{Acknowledgements}

The current study was funded by the Conacyt (grant no. BASICA-CB2010-01-155082, SALUD-2012-16933 and SALUD-2010-C01-86996).

\section{References}

1. Guzman MG, Halstead SB, Artsob H, Buchy P, Farrar J, Gubler DJ, Hunsperger E, Kroeger A, Margolis HS, Martínez E, et al: Dengue: A continuing global threat. Nat Rev Microbiol 8 (12 Suppl): S7-S16, 2010.

2. Henchal EA and Putnak JR: The dengue viruses. Clin Microbiol Rev 3: 376-396, 1990.
3. Halstead SB, Nimmannitya S and Cohen SN: Observations related to pathogenesis of dengue haemorrhagic fever. IV. Relation of disease severity to antibody response and virus recovered. Yale J Biol Med 42: 311-328, 1970.

4. Gibbons RV, Kalanarooj S, Jarman RG, Nisalak A, Vaughn DW, Endy TP, Mammen MP Jr and Srikiatkhachorn A: Analysis of repeat hospital admissions for dengue to estimate the frequency of third or fourth dengue infections resulting in admissions and dengue hemorrhagic fever, and serotype sequences. Am J Trop Med Hyg 77: 910-913, 2007.

5. Poh MK, Shui G, Xie X, Shi PY, Wenk MR and Gu F: U18666A, an intra-cellular cholesterol transport inhibitor, inhibits dengue virus entry and replication. Antiviral Res 93: 191-198, 2012.

6. Lee CJ, Lin HR, Liao CL and Lin YL: Cholesterol effectively blocks entry of flavivirus. J Virol 82: 6470-6480, 2008.

7. Chazal $\mathrm{N}$ and Gerlier D: Virus entry, assembly, budding and membrane rafts. Microbiol Mol Biol Rev 67: 226-237, 2003.

8. Rothwell C, Lebreton A, Young Ng C, Lim JY, Liu W, Vasudevan S, Labow M, Gu F and Gaither LA: Cholesterol biosynthesis modulation regulates dengue viral replication. Virology 389: 8-19, 2009.

9. Carro AC and Damonte EB: Requirement of cholesterol in the viral envelop for dengue virus infection. Virus Res 174: 78-87, 2013.

10. Martínez-Gutierrez M, Castellanos JE and Gallego-Gómez J: Statins reduce dengue virus production via decreased virion assembly. Intervirology 54: 202-216, 2011.

11. Umashankar M, Sánchez-San Martín C, Liao M, Reilly B, Guo A, Taylor G and Kielian M: Differential cholesterol binding by class II fusion proteins determines membrane fusion properties. J Virol 82: 9245-9253, 2008.

12. Ali N, Allam H, Bader T, May R, Basalingappa KM, Berry WL, Chandrakesan P, Qu D, Weygant N, Bronze MS, et al: Fluvastatin interferes with hepatitis $\mathrm{C}$ virus replication via microtubule bundling an a doublecortin-like kinase-mediated mechanism. PloS One 8: e80304, 2013.

13. Liu SY, Aliyari R, Chikere K, Li G, Marsden MD, Pernet O, Guo H, Nusbaum R, Zack JA, et al: Interferon-inducible cholesterol-25-hydroxylase broadly inhibits viral entry by production of 25-hydroxycholesterol. Immunity 38: 92-105, 2013.

14. Dai J, Pan W and Wang P: ISG15 facilitates cellular antiviral response to dengue and west nile virus infection in vitro. Virol J 8: 468, 2011.

15. Blanc M, Hsieh WY, Robertson KA, Kropp KA, Forster T, Shui G, Lacaze P, Watterson S, Grifitts SJ, Spann NJ, et al: The transcription factor STAT-1 couples macrophage synthesis of 25-hydroxycholesterol to the interferon antiviral response. Immunity 38: 106-118, 2013.

16. Reyes-Del Valle J, Chávez-Salinas S, Medina F and Del Angel RM: Heat shock protein 90 and heat shock protein 70 are components of dengue virus receptor complex in human cells. J Virol 79: 4557-4567, 2005.

17. Soto-Acosta R, Mosso C, Cervantes-Salazar M,Puerta-Guardo H, Medina F, Favari L, Ludert JE and del Angel RM: The increase in cholesterol levels at early stages after dengue virus infection correlates with an augment in LDL particle uptake and HMG-CoA reductase activity. Virology 442: 132-147, 2013.

18. Peña $J$ and Harris E: Early dengue virus protein synthesis induces extensive rearrangement of the endoplasmic reticulum independent of the UPR and SREBP-2 pathway. PloS One 7: e38202, 2012.

19. Gudleski-O'Regan N, Greco TM, Cristea IM and Shenk T: Increased expression of LDL receptor-related protein 1 during human cytomegalovirus infection reduces virion cholesterol and infectivity. Cell Host Microbe 12: 86-96, 2012.

20. Guabiraba R, Marques RE, Besnard AG, Fagundes CT, Souza DG, Ryffel B and Teixeira MM: Role of the chemokine receptors CCR1, CCR 2 and CCR4 in the pathogenesis of experimental dengue infection in mice. PloS One 5: e15680, 2010.

21. Reis SR, Sampaio AL, Henriques Md, Gandini M, Azeredo EL and Kubelka CF: An in vitro model for dengue virus infection that exhibits human monocyte infection, multiple cytokine production and dexamethasone immunomodulation. Mem Inst Oswaldo Cruz 102: 983-990, 2007. 